\title{
A diagnostic test of unawareness of bilateral motor task abilities in anosognosia for hemiplegia
}

\author{
I Nimmo-Smith, A J Marcel, R Tegnér
}

J Neurol Neurosurg Psychiatry 2005;76:1 167-1 169. doi: 10.1136/jnnp.2004.053744

\begin{abstract}
Anosognosia for hemiplegia (AHP) is conventionally defined/diagnosed by generic questions about awareness of limb plegia. However, unawareness of inability to perform tasks requiring bilateral use of limbs is more widespread and outlasts generic unawareness of plegia. Some patients consistently overestimate bilateral task ability. Our aim was to assess how well specific questions about bilateral task ability predict whether patients consistently overestimate their abilities. Six statistical indices were calculated to rank the questions for predictiveness of consistency of overestimation of bilateral task ability. Overall, bimanual questions are better predictors than bipedal questions of consistent overestimation. Three bimanual and two bipedal questions had both sensitivity and specificity above $80 \%$. On the basis of accuracy and discriminability, one bimanual and one bipedal question that performed maximally could be used for a quick bedside heuristic index. For a more thorough diagnostic, especially for research, five bimanual and two bipedal questions were good predictors, and should be used. For both purposes, such tests should be given in combination with conventional generic questions assessing awareness of limb plegia, since the two kinds of question reflect different kinds of unawareness of motor incapacity.
\end{abstract}

$\mathrm{T}$ aim of this paper is to report predictiveness statistics on a test of unawareness of motor task abilities in anosognosia for hemiplegia (AHP). Anosognosia refers to unawareness or denial of a deficit following brain damage ${ }^{12}$ and is the greatest impediment to rehabilitation. ${ }^{3}$ However, despite a century of research, AHP remains poorly understood. ${ }^{45}$ One basic problem lies in the diagnostic classification itself of patients. Almost all classification, indeed the standard criterion, has been based on conversational content or responses to questions, ${ }^{67}$ or, at best, formalisation of this in a structured interview. ${ }^{8}$

However, we have found ${ }^{4}$ that many patients who on these criteria are aware of having a plegia or have recovered such awareness, remain unaware of its consequences or entailments. That is, quite apart from any tendency to attempt impossible tasks, such patients are unaware on questioning of their inability to execute tasks that are bilateral and require use of the plegic $\operatorname{limb}(\mathrm{s})$. This latter unawareness can outlast the former unawareness by a significant period. We used a set of questions involving estimation of ability to carry out unimanual, bimanual, and bipedal tasks (see Appendix B in Marcel et $a l^{4}$ ). The purpose of the present analysis was to assess performance of the bilateral questions so that they can be used as a concise but predictive test for awareness of lack of abilities that can be adapted to either quick bedside diagnosis or as a research tool. Specifically, our aim was to calculate how well each question predicts whether a patient consistently overestimates their bilateral task abilities.

\section{PARTICIPANTS}

The original study was carried out on 42 right brain damaged (RBD) and 22 left brain damaged (LBD) stroke patients. For exclusion criteria, including aphasia, and for discussion of this issue, see Marcel et al. ${ }^{4}$ At the time of testing all had severe weakness of the arm, 35 RBD and 16 LBD patients had severe weakness of the leg, and the rest had moderate weakness in the leg. Interview questions about their present condition ("Why are you in hospital?", "Can you move your arms/legs?", etc) were adapted from Anderson and Tranel ${ }^{8}$ ( see Appendix A in Marcel $e t \mathrm{al}^{4}$ ). Only five RBD patients were unaware of why they were in hospital and that they had a hemiplegia. When specifically asked about motor function in arms and legs, 11/42 RBD and 2/22 LBD patients were unaware of a motor deficit.

\section{BILATERAL TASK QUESTIONS AND ORIGINAL PERFORMANCE}

The tasks enquired of regarding current bilateral task abilities are listed in table 1 . The present analysis was conducted on questions of the form: "In your present state how well, compared with your normal ability, can you tie a knot?, If you can do it as well as usual, say 'ten'.", "If you cannot do it at all, say 'nought'.". An estimate of 5 or more was counted as overestimation on that question. Some patients consistently overestimated their abilities. Individuals were classified as consistent bimanual overestimators if they overestimated five or more of these eight tasks, and consistent bipedal overestimators if they overestimated three or more of these four tasks. Table 1 shows the numbers of LBD and RBD patients who were and were not anosognosic on conventional criteria and who overestimated no bimanual tasks, up to four, and five or more. There was a small double dissociation in that a few patients unaware on conventional questions were not consistent overestimators.

Hardly any LBD patients overestimated their task abilities, except on three tasks that they had plausibly encountered in occupational therapy (see table 5 in Marcel et $a l^{4}$ ). Only one was a consistent overestimator on bimanual tasks and none were consistent overestimators on bipedal tasks. Of the RBD patients, the percentage overestimating each task ranged between $17 \%$ and $81 \%$. Twenty two were consistent overestimators on bimanual tasks and five of these were consistent overestimators on bipedal tasks. What makes task ability estimates of particular interest is the fact that 22 patients consistently overestimated their ability on bimanual tasks, while only five of them were unaware of their hemiplegia and only 11 of them were unaware of a motor deficit when specifically asked about the relevant limb. The

Abbreviations: AHP, anosognosia for hemiplegia; LBD, left brain damaged; RBD, right brain damaged 
Table 1 Number of bimanual tasks overestimated by LBD and RBD patients who were anosognosic or not on conventional criteria

\begin{tabular}{lllcc}
\hline & & \multicolumn{2}{c}{ Number of bimanual tasks overestimated } \\
\cline { 3 - 5 } & $\begin{array}{l}\text { Unawareness } \\
\text { of hemiplegia }\end{array}$ & 0 & $\mathbf{1 - 4}$ & $\begin{array}{l}\mathbf{5 - 8} \text { (consistent } \\
\text { overestimators) }\end{array}$ \\
\hline \multirow{2}{*}{ LBD } & Unaware & 0 & 2 & 0 \\
& Aware & 4 & 15 & 1 \\
& UnD & 1 & 2 & 10 \\
& Unaware & 4 & 13 & 12 \\
\hline
\end{tabular}

Unawareness of hemiplegia: unawareness on either (a) "Why are you in hospital?/What is wrong with you?", or (b) "Do you have any problems moving/any weakness in your arms?/legs? (This one? That one?)".

responses of the RBD patients constitute the data of the present analysis. This was performed to reveal how well, on various criteria, each of the eight bimanual and four bipedal tasks enquired of predict consistent overestimation.

\section{DESIGN AND METHOD}

The focus of interest is in the relationship between (a) a binary classification $\mathrm{O}$ (that is, is a person a consistent overestimator $(\mathrm{O}+)$ or not $(\mathrm{O}-)$ of bilateral task ability) that summarises performance across a range of questions, and (b) each question Q (for example, estimation of current ability to tie a knot) that indicates awareness of ability on one of the constituent tasks. The aim was to assess the extent to which overestimation $(\mathrm{Q}+)$ or non-overestimation $(\mathrm{Q}-$ ) on $\mathrm{Q}$ can be used as a predictor for the presence $(\mathrm{O}+)$ or absence $(\mathrm{O}-)$ of $\mathrm{O}$.

There are a variety of indices which quantify the ability of a binary question $\mathrm{Q}$ to indicate a binary state $\mathrm{O}$. The multiplicity of these indices reflects the fact that "there is no one simple index which enables us to compare different tests in all the ways we would like". ${ }^{\prime}$ This paper uses six indices (see table 2) in combination to rank the predictiveness of the questions. The criterion used was to select questions for which sensitivity and specificity are both greater than $80 \%$, and then to rank these by predictive accuracy (or equivalently by discriminability). ${ }^{9}{ }^{10}$

\section{RESULTS AND DISCUSSION}

Table 2 shows the results of the analysis. They are best summarised in terms of two ways in which they can be used. First, as a bedside heuristic, one needs one or two indicative questions. Of the bimanual questions, five ("clap", "shuffle", "catch", "wash", "cut") performed with sensitivity and specificity below $80 \%$. Of the remaining three, the best in terms of predictive accuracy and discriminability is "row a boat", followed by "tie a knot" and "unscrew a bottle". On this basis the "row" question could be used alone or in conjunction with "tie" and/or "unscrew". Of the bipedal questions, "jump" is consistently maximal for all indices, followed by "climb". On this basis the "jump" question could be used alone or with "climb".

Second, for research or to classify consistent overestimators, one needs a criterial set of questions. Three bimanual questions ("catch", "wash", "cut") are poor predictors in terms of predictive accuracy and discriminability, while the remaining five ("tie", "row", "unscrew", "clap", "shuffle") are good predictors (predictive accuracy greater than $80 \%$ ). By the same criterion, two bipedal questions ("jump", "climb") are good predictors and the other two are rather poor. In our original paper our criterion for consistent overestimation was overestimation on $5 / 8$ bimanual questions and on $3 / 4$ bipedal questions. If in total five bimanual and two bipedal questions are used, what classifying criteria are appropriate? Since the five bimanual questions are all good predictors, they could be used in order of weighting according to their predictability with a criterion (of consistent overestimation) of $4 / 5$. Since only two bipedal questions are relatively good predictors, we conclude that overestimation on both should be criterial for consistent bipedal overestimation.

Of the questions used, bipedal questions are worse predictors of consistent overestimation than bimanual questions. This has both diagnostic and practical importance: overestimation of bipedal ability can cause more severe

Table 2 Statistical indices of predictiveness of overestimation of current ability on a set of bimanual and bipedal tasks for consistent bimanual and bipedal overestimation

\begin{tabular}{|c|c|c|c|c|c|c|c|}
\hline & Tasks & Sensitivity (\%) & Specificity (\%) & PPV (\%) & NPV (\%) & Accuracy (\%) & Discriminability $\left(d^{\prime}\right)$ \\
\hline $\begin{array}{l}\text { Consistent bimanual } \\
\text { overestimation (prevalence } \\
52.4 \% \text { ) as predicted by: }\end{array}$ & $\begin{array}{l}\text { Tie a knot? } \\
\text { Clap hands? } \\
\text { Shuffle cards? } \\
\text { Row a boat? } \\
\text { Catch a ball? } \\
\text { Wash hands? } \\
\text { Unscrew bottle? } \\
\text { Cut bread? }\end{array}$ & $\begin{array}{c}95.5 \\
77.3 \\
66.7 \\
95.0 \\
100 \\
100 \\
81.8 \\
90.9\end{array}$ & $\begin{array}{c}85.0 \\
90.0 \\
100 \\
95.0 \\
40.0 \\
55.0 \\
90.0 \\
60.0\end{array}$ & $\begin{array}{c}87.5 \\
89.5 \\
100 \\
95.0 \\
64.7 \\
71.0 \\
90.0 \\
71.4\end{array}$ & $\begin{array}{c}94.4 \\
78.3 \\
73.1 \\
95.0 \\
100 \\
100 \\
81.8 \\
85.7\end{array}$ & $\begin{array}{l}90.5 \\
83.3 \\
82.5 \\
95.0 \\
71.4 \\
78.6 \\
85.7 \\
76.2\end{array}$ & $\begin{array}{l}2.49 \\
1.89 \\
2.42 \\
2.93 \\
1.96 \\
2.27 \\
2.04 \\
1.51\end{array}$ \\
\hline $\begin{array}{l}\text { Consistent bipedal } \\
\text { overestimation (prevalence } \\
11.9 \% \text { ) as predicted by: }\end{array}$ & $\begin{array}{l}\text { Ride a bicycle? } \\
\text { Climb a ladder? } \\
\text { Jump? } \\
\text { Walk? }\end{array}$ & $\begin{array}{l}60.0 \\
80.0 \\
80.0 \\
80.0\end{array}$ & $\begin{array}{l}70.3 \\
89.2 \\
91.9 \\
70.3\end{array}$ & $\begin{array}{l}21.4 \\
50.0 \\
57.1 \\
26.7\end{array}$ & $\begin{array}{l}92.9 \\
97.1 \\
97.1 \\
96.3\end{array}$ & $\begin{array}{l}69.0 \\
88.1 \\
90.5 \\
71.4\end{array}$ & $\begin{array}{l}0.64 \\
1.72 \\
1.89 \\
1.03\end{array}$ \\
\hline
\end{tabular}

Sensitivity (of $Q$ to $O$ ): the proportion of consistent overestimators $(O+)$ who overestimate task $Q$, that is, $P(Q+\mid O+)$. Another name for this is the hit rate or true positive rate.

Specificity (of $Q$ for $O$ ): the proportion of patients that are not consistent overestimators who do not overestimate on $Q, P(Q-\mid O-)$. It is equal to 1 minus the false alarm rate of $Q$, that is, $P(Q+\mid O-)$. Another name for specificity is the true negative rate.

PPV: the positive predictive value of $Q$ for $O$. This is the proportion of patients that overestimate task $Q$ who would be correctly identified as $O+$ on the basis of observing $Q+$, that is, $P(O+\mid Q+)$.

NPV: the negative predictive value of $Q$ for $O$. This is the proportion of patients that do not overestimate task $Q$ who are not consistent overestimators, that is, $P(O-\mid Q-)$.

Accuracy: the predictive accuracy of $Q$ for $O$. This is the proportion of patients for which the outcome of $Q$ matches the state of $O$, that is, the proportion that are correctly classified by $Q$ with respect to $O$.

Discriminability: D-prime $\left(d^{\prime}\right)$, the classical signal detection theory index of detectability. It estimates how far the $\mathrm{O}-$ and $\mathrm{O}+$ patients are separated on the basis of Q- and Q+.

Prevalence: the proportion of patients who are consistent overestimators, that is, $\mathrm{P}(\mathrm{O}+)$. 
accidents. The disparity in predictability may be due partly to the fewer bipedal questions and therefore a less reliable criterion. It may also be due to the relation between plegia and unawareness of it.

In summary, a bedside test should include both (a) conventional generic questions (for example, "What is wrong with you?", "Can you move both your arms/legs normally?"; see Anderson and Tranel $^{8}$ and our adaptation ${ }^{4}$ ), and (b) one bimanual task question ("How well could you row a boat in your present condition compared to normal?") and one bipedal task question ("How well could you jump ... ?"). For research purposes and a more thorough index, we recommend adding to the conventional generic questions the five bimanual and two bipedal questions specified above. While the generic questions do diagnose AHP, the latter kind of question reveals a further kind of unawareness of motor incapacity (as implied by the double dissociation shown in table 1) and is more relevant to management and rehabilitation.

\section{Authors' affiliations}

I Nimmo-Smith, A J Marcel, Medical Research Council Cognition and Brain Sciences Unit, Cambridge, UK

R Tegnér, Department of Clinical Neuroscience, Neurology Section, Karolinska Institute, Stockholm, Sweden

Competing interests: none declared

Correspondence to: Dr A J Marcel, MRC Cognition and Brain Sciences Unit, 15 Chaucer Road, Cambridge CB2 2EF, UK; tony.marcel@ mrc-cbu.cam.ac.uk
Correspondence may also be addressed to $\operatorname{Dr} I$ Nimmo-Smith at ian.nimmo-smith@mrc-cbu.cam.ac.uk or to R Tegnér at richard.tegner@ telia.com

Received 9 September 2004

Revised version received 26 October 2004

Accepted 19 November 2004

\section{REFERENCES}

1 Bisiach E, Geminiani G. Anosognosia related to hemiplegia and hemianopia. In: Prigatano GP, Schacter DL, eds. Awareness of deficit after brain injury. New York: Oxford University Press, 1991:17-39.

2 Weinstein EA. Anosognosia and denial of illness. In: Prigatano GP, Schacter DL, eds. Awareness of deficit after brain injury. New York: Oxford University Press, 1991:240-57.

3 Gialanella B, Mattioli F. Anosognosia and extrapersonal neglect as predictors of functional recovery following right hemisphere stroke. Neuropsychol Rehabil 1992;2:169-78.

4 Marcel AJ, Tegnér R, Nimmo-Smith I. Anosognosia for plegia: specificity, extension, partiality, and disunity of bodily unawareness. Cortex 2004;40:19-40.

5 Vuilleumier $\mathrm{P}$. Anosognosia: the neurology of beliefs and uncertainties. Cortex 2004;40:9-17.

6 Prigatano GP, Schacter DL. Awareness of deficit after brain injury. New York: Oxford University Press, 1991.

7 Bisiach E, Vallar G, Perani D, et al. Unawareness of disease following lesions of the right hemisphere: anosognosia for hemiplegia and anosognosia for hemianopia. Neuropsychologia 1986;24:471-82.

8 Anderson SW, Tranel D. Awareness of disease states following cerebral infarction, dementia, and head trauma: standardized assessment. Clin Neuropsychol 1989;3:327-39.

9 Bland M. An introduction to medical statistics, 3rd ed. Oxford: Oxford University Press, 2000:275-9.

10 Macmillan NA, Creelman CD. Detection theory: a user's guide. Cambridge: Cambridge University Press, 1991:7-25. 